\title{
Spontaneous rupture of urinary bladder diverticulum with pseudo-acute renal failure
}

\author{
Chih-Wei Sung ${ }^{1} \cdot$ Chin-Chen Chang ${ }^{3} \cdot$ Shey-Ying Chen ${ }^{2} \cdot$ Wen-Pin Tseng ${ }^{2}$ (i)
}

Received: 8 January 2018 / Accepted: 23 January 2018 / Published online: 30 January 2018

c) SIMI 2018

\section{Case report}

A 36-year-old woman presented to the emergency department (ED) with sudden onset, post-micturition lower severe abdominal pain of 1 day duration. She had a history of left thyroid nodules and anemia of chronic disorder, but without any surgical history. There were no other associated symptoms such as chills, nausea, vomiting, diarrhea, constipation or hematuria. She also denied pregnancy, trauma or alcohol consumption.

On examination, she was fully conscious, and her vital signs were body temperature of $37.7{ }^{\circ} \mathrm{C}$, pulse rate of 100 beats per minute, blood pressure of $121 / 84 \mathrm{mmHg}$, respiratory rate of 20 breaths per minute and the oxygen saturation $99 \%$ on room air. Her abdomen was distended with diffuse rebound tenderness and hypoactive bowel sounds. The remainder of her systematic examination was unremarkable.

Results of biochemical analysis showed elevated serum levels of urea nitrogen (UN) and creatinine, indicative of acute renal failure (Table 1). Other laboratory examinations, including hemogram, urinalysis and coagulation test, were within the normal ranges. Ultrasound of the abdomen demonstrated ascites and mild distended urinary bladder without hydronephrosis. Diagnostic abdominal paracentesis confirmed the presence of transudative ascites with serum-ascites albumin gradient (SAAG) value greater than

Wen-Pin Tseng

endless0228@ntuh.gov.tw

1 Department of Emergency Medicine, National Taiwan University Hospital Hsin-Chu Branch, National Taiwan University College of Medicine, Hsinchu, Taiwan

2 Department of Emergency Medicine, National Taiwan University Medical College and Hospital, National Taiwan University, Taipei, Taiwan

3 Department of Medical Imaging, National Taiwan University Hospital, National Taiwan University College of Medicine, Taipei, Taiwan
3. Additionally, spontaneous peritonitis was excluded based on normal ascitic fluid polymorphonuclear leukocyte count. Non-contrast computed tomography (CT) of the abdomen and pelvis revealed urinary bladder diverticulum with peritoneal fluid accumulation, which suggested urinary leakage. We prescribed antibiotics and inserted a Foley catheter. Notably, the abdominal distension and tenderness began to resolve, and the serum urea nitrogen and creatinine levels dramatically decreased to a normal range within $16 \mathrm{~h}$ (Table 1), which indicated the possibility of ruptured urinary bladder causing urinary ascites and peritoneal irritation. A CT cystography showed extravasation of contrast into the peritoneal space and confirmed the diagnosis of ruptured urinary bladder diverticulum (Fig. 1). The urologist performed a cystoscopy and laparoscopic diverticulectomy after admission and identified a $5 \times 5 \mathrm{~cm}$ diverticulum at the left lateral wall near the bladder dome intraoperatively. The patient was discharged home on postoperative day 5 uneventfully.

\section{Discussion}

Spontaneous or non-traumatic rupture of the urinary bladder is a rare, but potentially life-threatening event in adult patients. This condition is associated with certain specific predisposing factors including binge alcohol drinking, pregnancy, infection, neurologic bladder, diabetes, benign prostatic hyperplasia and postpartum complications [1]. Nevertheless, rupture of a urinary bladder from diverticulum is extremely rare, and only few cases have been reported in the literature [2]. The etiologies of urinary bladder diverticulum rupture may originate from congenital anatomic abnormality of pathological urinary bladder wall weakness, but the majority are acquired, including: idiopathic spontaneous rupture of ongoing chronic obstructive diseases, frequent urine retention with increased bladder pressure, iatrogenic intraoperative injury, and radiotherapy for pelvic 
Table 1 Biochemical analysis in serum, urine and abdominal ascites

\begin{tabular}{llllllll}
\hline & \multicolumn{3}{l}{ First day of admission } & & & \multicolumn{2}{l}{ Second day of admission } \\
\cline { 2 - 3 } & Serum & Ascites & Urine & & Serum & Ascites & Urine \\
\hline $\mathrm{UN}(\mathrm{mg} / \mathrm{dL})$ & 34.5 & 89 & 1429.8 & & \\
$\mathrm{Cr}(\mathrm{mg} / \mathrm{dL})$ & 2.5 & 17.1 & 240 & & 0.7 & \\
$\mathrm{Na}(\mathrm{mmole} / \mathrm{L})$ & 135 & 137 & 135 & & 135 & \\
$\mathrm{~K}(\mathrm{mmole} / \mathrm{L})$ & 4.7 & 7.0 & 63 & & 3.9 & \\
$\mathrm{Cl}(\mathrm{mmole} / \mathrm{L})$ & & 109 & & & & \\
$\mathrm{Alb}(\mathrm{g} / \mathrm{dL})$ & 4.1 & $<1.5$ & & & & \\
\hline
\end{tabular}

$U N$ urea nitrogen, $\mathrm{Cr}$ creatinine, $\mathrm{Na}$ sodium, $\mathrm{K}$ potassium, $\mathrm{Cl}$ chloride, $\mathrm{Alb}$ albumin malignancies [3]. Most patients present with acute lower abdominal pain, dysuria, difficulty voiding and hematuria. Patients can also present as acute renal failure due to decreased urine output, elevated serum urea nitrogen and creatinine level, which actually results from peritoneal resorption of urea nitrogen and creatinine from urinary ascites [4]. Clinicians may easily misdiagnose this rare but potentially catastrophic disease as intraabdominal infection from the gastrointestinal tract because of the apparent peritoneal irritation, elevated serum levels of UN and creatinine level, misdirecting clinicians to manage acute renal failure and peritonitis, but causing them to ignore the nature of urinary ascites from the ruptured bladder.

Our patient presented to the hospital with acute renal failure on laboratory examination. Rapidly increasing a previously normal serum UN and creatinine without an acute kidney injury is the key feature of urinary ascites. Impaired renal function caused by urinary ascites does not belong to the traditional classification of acute renal failure because the pathophysiology does not involve hypovolemic states, fluid sequestration, vascular ischemia, glomerular diseases, tubular necrosis or obstructive diseases. The mechanism is related to the reabsorption of creatinine in the urinary ascites via the peritoneal membrane. The elevated creatinine level originates from the concept of "reverse auto-dialysis" of the peritoneal membrane, which was first described in early 1990 [5]. It is a reverse form of continuous ambulatory peritoneal dialysis that systematically absorbs toxic metabolites. As a result, elevated serum UN and creatinine mimic renal failure despite normally functioning kidneys. It is described as "pseudo-renal failure", a status of laboratory abnormalities of acute kidney injury in the setting of normal kidney function from urinary ascites. Clinicians should distinguish pseudo-renal failure from true renal failure based on laboratory analysis of peritoneal fluid, which has higher levels of UN, creatinine and potassium levels than those in serum levels, as demonstrated in our patient. Unnecessary renal replacement therapy can be avoided with prompt diagnosis and proper emergent management.

The gold standard for diagnosis of urinary bladder diverticulum rupture is retrograde cystography or CT urography. Foley catheter drainage has become a standard management initially, which improves the patient's symptoms and normalizes the serum levels of creatinine in cases of urinary bladder rupture [6]. The serum levels of creatinine usually return to a normal range in a short time. Our patient returned to a normal range $16 \mathrm{~h}$ after the insertion of a Foley catheter. Definitive treatment for intraperitoneal urinary bladder rupture typically requires surgical repair. Secondary bacterial peritonitis and sepsis is one of the major complications of a ruptured urinary bladder diverticulum with urinary ascites. Clinicians should promptly administer antibiotics to prevent this potential septic complication before the definite repair of the ruptured diverticulum and resolution of urinary ascites.

In conclusion, acute lower abdominal pain with rebound tenderness, difficulty in urination and oliguria should prompt clinicians the suspicion of urinary bladder rupture. Intraperitoneal urine leakage needs to be considered in the presence of acute renal failure, recurrent ascites and an elevated peritoneal fluid creatinine level higher than serum creatinine level. Clinicians should recognize urinary bladder rupture with urinary ascites as early as possible for a timely surgical intervention, minimizing metabolic and septic complications, and best treatment outcome of this potentially catastrophic disease. 

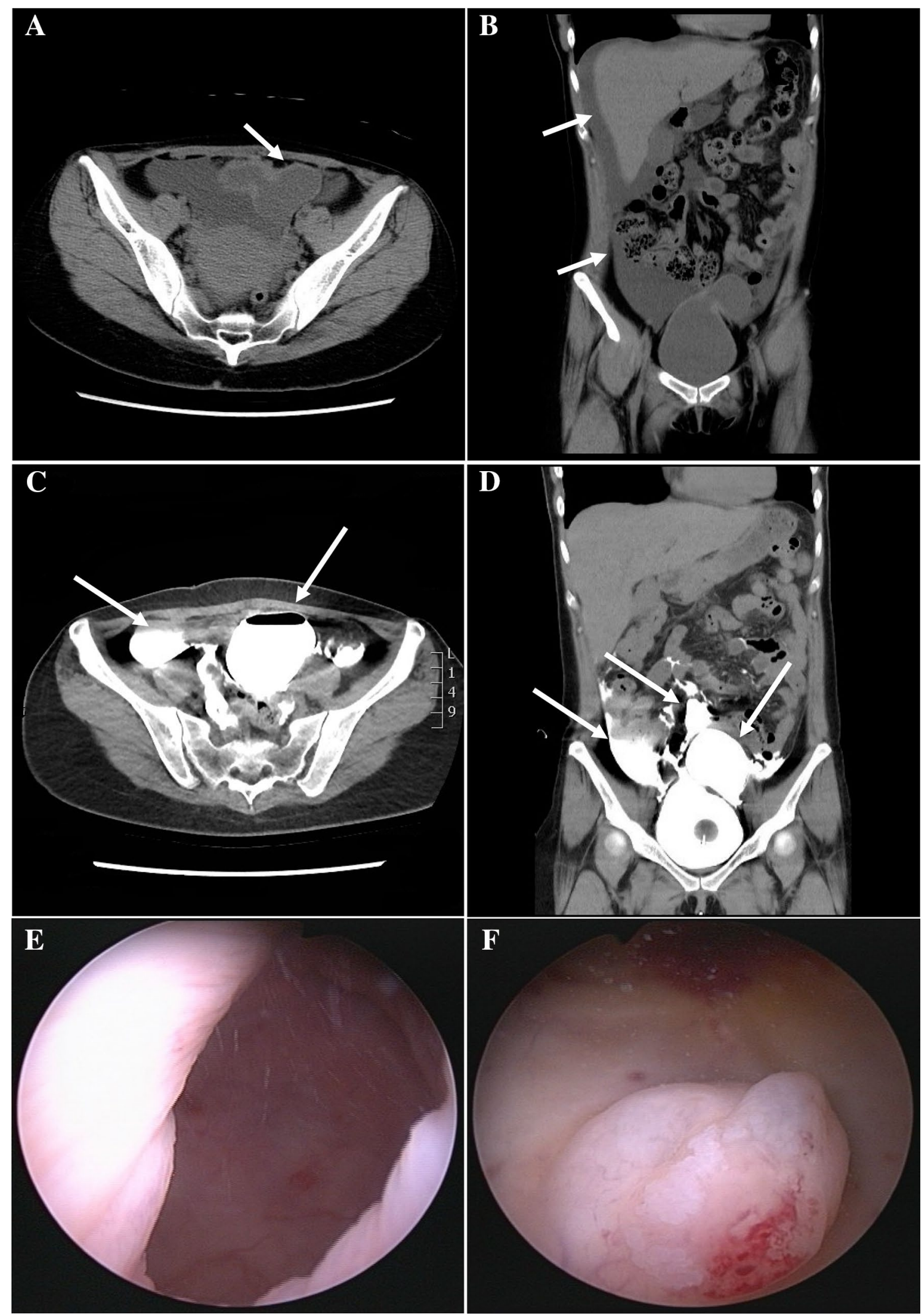

Fig. 1 Emergent CT scan of abdomen and pelvis shows a urinary bladder diverticulum and $\mathbf{b}$ ascites with peritoneal infiltration (arrows). c, $\mathbf{d}$ CT urography shows a significant bladder diverticulum on the left dome of the urinary bladder and the extravasation of contrast into peritoneal cavity, suggestive of rupture in transverse and coronary view, respectively. e Cystoscopy identified a $5 \times 5 \mathrm{~cm}$ bladder diverticulum at the left lateral wall near dome and $\mathbf{f}$ a protruding bladder diverticulum at the surface of the bladder before laparoscopic bladder diverticulectomy 


\section{Compliance with ethical standards}

\section{Conflict of interest None.}

Human and animal rights statement All procedures performed in studies involving human participants were in accordance with the ethical standards of the institutional or national research committee and with the 1964 Helsinki Declaration and its later amendments or comparable ethical standards. This article does not contain any studies with animals performed by any of the authors.

Informed consent For this type of study, formal consent is waived.
2. Leahy O, Grummet J (2013) Splash! The spontaneous rupture of a bladder diverticulum: a rare cause of an acute abdomen. ANZ J Surg 83(10):792-793. https://doi.org/10.1111/ans.12240

3. Silberman M, Jeanmonod R (2011) Bladder diverticulitis: a case report. Case Rep Emerg Med 2011:303498. https://doi. org/10.1155/2011/303498

4. Dees A, Kluchert SA, van Vliet AC (1990) Pseudo-renal failure associated with internal leakage of urine. Neth J Med 37(5-6):197-201

5. Oriot D, Gauthier F, Huault G (1992) Reverse peritoneal dialysis or acute renal failure. Clin Nephrol 37(4):216

6. Arnold WC, Redman JF, Seibert JJ (1986) Analysis of peritoneal fluid in urinary ascites. South Med J 79(5):591-594

\section{References}

1. Pradhan AA (2007) Spontaneous rupture of bladder: a rare clinical entity. Med J Armed Forces India 63(1):92-93. https://doi. org/10.1016/S0377-1237(07)80126-2 